\title{
Cervical Cancer in Pregnant Women: A Case Report
}

Mohamed Adnane Rhaidouni ${ }^{1 *}$, Yassine Outifa ${ }^{1}$, Mohamed Cheikh Abderrahmane ${ }^{1}$, Sofia Jayi ${ }^{1}$, Fatima Zohra Fdili Alaoui $^{1}$, Hikmat Chaara ${ }^{1}$, Moulay Abdelilah Melhouf ${ }^{1}$

${ }^{1}$ Sidi Mohamed Ben Abdellah University, Department of Gynecology - Obstetrics II, Hassan II Teaching Hospital, Fez Morocco

DOI: 10.36348/sijog.2021.v04i04.003 | Received: 26.02.2021 | Accepted: 01.04.2021 | Published: 11.04.2021

*Corresponding author: Mohamed Adnane Rhaidouni

\section{Abstract}

The process itself of staging of the disease during pregnancy and the treatment should comply with current standards, to the extent possible. Surgery and/or chemotherapy are considered to be fundamental methods of treatment for invasive cervical cancer during pregnancy. Tactics in the treatment of such patients is determined by a range of factors and depends on gestational age at the time of diagnosis, the stage of the disease, tumor size, as well as on the involvement of regional lymph nodes and the woman's desire to continue pregnancy. Case Description: A 32 year-old patient, grand multipara, admitted for spontaneous metrorrhagia of low abundance during a 7 month pregnancy (date of last menstrual period is inaccurate) for which she consulted in the private sector where she underwent a gynecological examination that showed a tumoral process of the cervix, and then a biopsy that revealed a cervical squamous cell carcinoma. The patient was then referred to our departement for further care; we decided to perform a C-section at 34 weeks of ammenorrhea, giving birth to a male infant with a birth weight of $2300 \mathrm{~g}$, and then a CRC. Conclusion: The incidence of cervical cancer in pregnancy is itself not very high, and the symptoms are easily confused with other diseases in pregnancy. During pregnancy, gynecological examination is limited, and therefore, the rate of misdiagnosis is higher. The treatment of cervical cancer during pregnancy is related to many factors, such as tumor size, pathological type, period of gestation, lymph node involvement, and patients' willingness to maintain pregnancy.

Keywords: Pregnancy, invasive cervical cancer, diagnosis.

Copyright () 2021 The Author(s): This is an open-access article distributed under the terms of the Creative Commons Attribution 4.0 International License (CC BY-NC 4.0) which permits unrestricted use, distribution, and reproduction in any medium for non-commercial use provided the original author and source are credited.

\section{INTRODUCTION}

The diagnosis of invasive cervical cancer during pregnancy is not always obvious. The incidence of dysplasia during pregnancy is estimated at $1 \%$ and that of the cervical cancer is at one in 10,000. Cervical cancer is one of the most commonly diagnosed cancers during pregnancy with the breast, with lymphomas and melanomas. However, the real incidence of the data remains unclear [1]. For patients with little follow-up, the beginning of pregnancy should be the occasion to take a cervical cytology if it has not been recently and to educate patients about the need of this surveillance [2]. The recommendation of cervical cytology in early pregnancy will increase the incidence of discovery of precancerous lesions, which is why it is important to define the guidelines. For cancers occurring during the pregnancy, the objectives are double and sometimes antagonistic: to achieve a management closer to that of patients who are not pregnant, i.e. do not subcontract because of pregnancy and if possible, keep the pregnancy. If previously an interruption pregnancy was often recommended during both pregnancy and childbirth. First quarters, recent publications report cases of preservation of pregnancy. This should not be to the detriment of the carcinological outcome [3].

\section{CASE PRESENTATION}

A 32-year-old female patient with an unremarkable medical history, from and living in Fez, G4P3 (3 living children/vaginal delivery), pregnant at 7 months (according to an inaccurate date of last menstrual period), characterized by the occurrence of light metrorrhagia in the first trimester, for which she consulted a gynecologist in the private practice (at 5 months of pregnancy) who has suspected a cervical tumor, and performed a biopsy, then referred the patient to our departement for further care. On general examination : WHO:1, slightly discolored conjunctiva 
The Gynecological examination revealed an ulcerative lesion of the cervix of 4 invading the upper $1 / 3$ of the vagina and proximally reaching the right parametrium. The cervical biopsy revealed a moderately differentiated epidermoid carcinoma with little keratinization and infiltrating character.

The Abdominopelvic MRI showed a cervical lesion process that measured $54.4 \mathrm{~mm}$ in transverse diameter, $43.8 \mathrm{~mm}$ in anteroposterior diameter and extended over $57 \mathrm{~mm}$ in height. It also infiltrates the mesorectum without endorectal or bladder extension. It is then classified IIb.

The chest X-ray was normal. The Abdominal ultrasound didn't show any further abdominal location. The Obstetrical ultrasound showed a progressive monofetal pregnancy, and a posterior placenta. The biometry was: p50 of 27-28 weeks of ammenorrhea, and the amniotic fluid of decreased quantity. The estimated weight was $1600 \mathrm{~g}$.

The hematological examination showed HB of 8 and urea/creatine of $0.12 / 6$. It was accordingly decided to discuss the extraction from 32-34 weeks, to perform a CRC after the extraction (If patient goes into labor = cesarean section) and to schedule a postoperative CT scan of the thoracic-abdominal-pelvic area.

The patient was hospitalized in our department for PRM in a 27-28 weeks of pregnancy for which she received antibiotic therapy, hemostatic treatment, martial supplementation and corticosteroid therapy (two doses at 28 and 32 weeks). A C-section was performed at 34 weeks with an extraction of a male newborn, Apgar 8/10, birth weight $2300 \mathrm{~g}$; the postoperative course was unremarkable. Subsequently, the patient was admitted to the departement of oncology for cervical radio-chemotherapy (CRC): she received a total dose of 46 Gy+boost with a concomitant chemotherapy of 4 courses and 04 sessions of curitherapy marked by a good tolerance and a good therapeutic response.

\section{DISCUSSION}

The most common gynecological malignant tumors in pregnancy are cervical cancer, accounting for $71.6 \%$, followed by ovarian malignant tumors, accounting for $7.0 \%$. The clinical manifestations of cervical cancer in pregnant women are related to the clinical stage and diameter of the tumor. Pregnancy with early cervical cancer mostly has no obvious clinical symptoms. However, a few symptomatic patients mostly show vaginal discharge with stench, purulent or bloody secretions, and vaginal irregular bleeding. Pregnancy with late cervical cancer mainly shows pain caused by tumors or chronic anemia caused by long- term irregular vaginal bleeding. Due to the fact that such patients are either pregnant or postpartum, the above symptoms are easily mistaken for other diseases during pregnancy or puerperium symptoms. Therefore, in pregnant patients and postpartum patients with vaginal bleeding, one should be very vigilant, and if necessary, gynecological examination and cervical exfoliation cytology screening are to be carried out.

Screening for pregnancy with cervical cancer also follows the "three- step model", namely, cervical cytology, colposcopy, and cervical biopsy.Cervical cytology is the first choice for rapid diagnosis of cervical cancer. The test does not pose a threat to mothers and children throughout pregnancy. Previous studies have shownthat the accuracy of cervical cytological diagnosis in pregnancy is similar to that in nonpregnancy[4,5]. However, recent studies have shown that changes in maternal estrogen and progesterone levels lead to glandular hyperplasia of cervical mucosa, migration of squamous- columnar junction, active proliferation of basal cells, irregular cell morphology, and enlargement of nuclei, which are easily misdiagnosed as highly squamous intraepithelial lesions or even invasive cancer[4, 6]. In view of the specificity of the cervix during pregnancy, it is recommended that cervical cytology smears be made by experienced pathologists who can then examine and make a conclusion on the film to reduce misdiagnosis [5]. The cervical image under colposcopy is often difficult to identify because of the change of maternal hormone level during pregnancy. Therefore, colposcopy is better to be undertaken within the first and second trimesters of pregnancy. If the early colposcopy is not satisfactory, it can be repeated after 20 weeks of pregnancy.

Analysis of prognosis of cervical cancer during pregnancy shows no negative impact of pregnancy on the outcome of patients;therefore, pregnancy-preserving management should be considered initially. The different treatment options based on stage and gestational age at diagnosis.

Pregnancy-preserving management. A cone biopsy may be used to treat stage IA1 tumors without lymphovascular space invasion.For stage IA1 with lymphovascular space invasion, IA2 and IB1, staging lymphadenectomy should be performed as a first step.This can be safely done up to the 22nd week of gestation. After the 22nd week of gestation, delayed treatment after delivery with regular follow-up could be initiated. Alternatively, neoadjuvant chemotherapy (NACT) could be used to control the disease.In stage IB2 less than the 22nd week of gestation, two options are available: (i) pelvic lymphadenectomy as a first step followedby either chemotherapy or follow-up, and (ii) NACT and subsequent surgical staging of the disease after downstaging the tumor.In the case of positive nodes (including micro metastases), we recommend termination of pregnancy. However, the panel believes that for those patients who refuse this option, 
chemotherapy could be considered. In these instances, patients should be informed of the possible negative impact on the prognosis and the lack of available data. Follow-up of IB1, IB2 and IB3 tumors after staging lymphadenectomy has been described in a systematic review of Morice et al. [4], who collected 76 patients with a median follow-up of 37.5 months (mean 16 weeks of delay) and showed excellent oncological outcome. After the 22nd week of gestation, only NACT is an option.

In stage IB3 (according to the new FIGO 2018 classification [7]), the only pregnancy-preserving option is the application of NACT, although its efficiency has only been investigated in a small number of trials, and further research is warranted [8].The role of staging lymphadenectomy is controversial [9]. Follow-up without therapy in such cases is likely to compromise the prognosis and is thus not recommended.

With increasing gestational age, a delay of definitive treatment is more commonly used, though NACT (until the 34-35th week of gestation) will prolong the duration of pregnancy until term delivery.

Pregnancy nonpreserving management. Pregnancy nonpreserving management is chosen in advanced disease (stage IIB or higher or lymph node metastases) or in cases when the patient chooses not to preserve her pregnancy (based on local legislation and usually until the 24th week of gestation). Treatment is thus planned without intention to preserve the fetus. In case of an operable disease (IA2-IB2), a radical hysterectomy with fetus in utero (during the first- or early-second trimester) or after hysterotomy (during the late second trimester) can be performed. In IB3 and higher stages, during first trimester chemoradiation can be applied with the fetus in utero (the death of the fetus occurs within few days), while during second trimester a hysterotomy as a first step is advised. This reduces the risk of obstetrical complications (bleeding, rupture of the cervix, diffuse intravascular coagulation) and psychological impact on the patient.Alternatively, before chemoradiotherapy is initiated, feticide can be considered for ethical and psychological reasons [10].

Cesarean section is the preferred method for delivering fetuses with giant cervical tumors. Vaginal delivery carries risks of vaginal laceration, massive hemorrhage at scar incision, and metastasis of tumors. When the tumors are locally advanced, transverse cesarean section should be avoided because of the risk of cutting or tearing the tumors. Classic vertical incision can reduce bleeding and avoid damaging the blood vessels of tumors. Postoperative placenta should be sent for pathological examination to determine whether there is any metastasis. The second international consensus issued by the International Association of Gynecological Oncology in 2014 pointed out that delivery could be postponed to full- term pregnancy (>37 weeks), but premature delivery would inevitably occur in some patients because of tumor progression or need of radiotherapy. Neonatologists should discuss the timing of delivery together at this time.

The effect of chemotherapy on the fetus depends on the dose of drugs transferred to the fetus by pregnant women receiving chemotherapy during pregnancy. Calsteren et al. [11] studied placental transport of chemotherapeutic drugs commonly used in pregnant baboon models.

The results showed that the average concentration of carboplatin in baboon fetal plasma was $57.5 \%$ of the maternal body; in addition, the concentration of paclitaxel in fetal umbilical cord blood was $15 \%$ of the maternal body after 3 hours of paclitaxel infusion; after transfusion of docetaxel, the concentration of docetaxel in fetal umbilical cord blood was $5 \%-50 \%$ of maternal plasma, while after 26 hours, the concentration of both was high. The transplacental transmission rate of trastuzumab decreased from $85 \%$ to $3 \%$ at 2 and 26 hours after trastuzumab injection. Kohler et al. [12] suggested that there might be a platinum placental filtration mechanism because the platinum concentrations in fetal cord blood and amniotic fluid were $23 \%-65 \%$ and $11 \%-24 \%$ of maternal blood, respectively. Chemotherapy can directly act on the growing fetus, or indirectly act on the growing fetus through the placenta.29, 30 After the development of fetal organs, chemotherapy can affect fetal eyes, genitals, hematopoietic system, and central nervous system.29 Chemotherapy- induced suppression of maternal and fetal bone marrow can also lead to anemia, which in turn affects fetal growth [13].

\section{CONCLUSION}

The clinical manifestations of pregnancy complicated withcervical cancer are atypical, easily confused with pregnancy diseases, easily concealed by pregnancy status, and difficult to diagnose. Prenatal examinations are often neglected by pregnant women, which make it difficult to detect tumors. The effective treatment to women with pregnancy complicated with cervical cancer is mainly aimed at safeguarding both maternal and fetal health. Because of the pregnancy, it is not always easy to diagnose and treat such women, and any interventions on behalf of physicians are limited in such cases.

\section{REFERENCES}

1. Duggan, B., Muderspach, L. I., Roman, L. D., Curtin, J. P., d'Ablaing 3rd, G., \& Morrow, C. P. (1993). Cervical cancer in pregnancy: reporting on planned delay in therapy. Obstetrics and gynecology, 82(4 Pt 1), 598-602.

2. Moran, B. J., Yano, H., Al Zahir, N., \& Farquharson, M. (2007). Conflicting priorities in 
surgical intervention for cancer in pregnancy. The lancet oncology, 8(6), 536-544.

3. Stensheim, H., Møller, B., Van Dijk, T., \& Fosså, S. D. (2009). Cause-specific survival for women diagnosed with cancer during pregnancy or lactation: a registry-based cohort study. Journal of Clinical Oncology, 27(1), 45-51.

4. Morice, P., Uzan, C., Gouy, S., Verschraegen, C., \& Haie-Meder, C. (2012). Gynaecological cancers in pregnancy. The Lancet, 379(9815), 558-569.

5. Morimura, Y., Fujimori, K., Soeda, S., Hashimoto, T., Takano, Y., Yamada, H., ... \& Sato, A. (2002). CERVICAL CYTOLOGY DURING PREGNANCY - Comparison with non-pregnant women and management of pregnant women with abnormal cytology-. Fukushima journal of medical science, 48(1), 27-37.

6. Shiping, Z., \& Lushan, Z. Q. (2018). Progress in diagnosis and treatment of pregnancy complicated with cervical cancer. J Pract Med, 25(4), 400-402.

7. Bhatla, N., Aoki, D., Sharma, D. N., \& Sankaranarayanan, R. (2018). Cancer of the cervix uteri. International journal of gynecology \& obstetrics, 143, 22-36.

8. Rydzewska, L., Tierney, J., Vale, C. L., \& Symonds, P. R. (2012). Neoadjuvant chemotherapy plus surgery versus surgery for cervical cancer. Cochrane Database of Systematic Reviews, (12).

9. Cibula, D., Pötter, R., Planchamp, F., AvallLundqvist, E., Fischerova, D., Haie-Meder, C., ... \& Raspollini, M. R. (2018). The European Society of Gynaecological Oncology/European Society for Radiotherapy and Oncology/European Society of Pathology guidelines for the management of patients with cervical cancer. Virchows Archiv, 472(6), 919-936.

10. Sood, A. K., Sorosky, J. I., Mayr, N., Krogman, S., Anderson, B., Buller, R. E., \& Hussey, D. H. (1997). Radiotherapeutic management of cervical carcinoma that complicates pregnancy. Cancer: Interdisciplinary International Journal of the American Cancer Society, 80(6), 1073-1078.

11. Van Calsteren, K., Verbesselt, R., Devlieger, R., De Catte, L., Chai, D. C., Van Bree, R., ... \& Amant, F. (2010). Transplacental transfer of paclitaxel, docetaxel, carboplatin, and trastuzumab in a baboon model. International Journal of Gynecologic Cancer, 20(9).

12. Köhler, C., Oppelt, P., Favero, G., Morgenstern, B., Runnebaum, I., Tsunoda, A., ... \& Marnitz, S. (2015). How much platinum passes the placental barrier? Analysis of platinum applications in 21 patients with cervical cancer during pregnancy. American journal of obstetrics and gynecology, 213(2), 206-e1.

13. Child Health Epidemiology Reference Group Kozuki Naoko Lee Anne C. Katz Joanne jkatz@ jhsph. edu Author disclosures: N. Kozuki, AC Lee, and J. Katz, no conflicts of interest. (2012). Moderate to severe, but not mild, maternal anemia is associated with increased risk of small-forgestational-age outcomes. The Journal of nutrition, 142(2), 358-362. 